ANÁLISIS [] ISSN: 0120-8454 [ Vol. 49 / No. 90 [ Bogotá, ene.-jun. / 2017 [ pp. 111-134.

\title{
Estudio sobre el estado de la literatura de la cultura organizacional en la administración pública desde el enfoque de análisis de contenidos*
}

\author{
Rafael Vergara Varela** \\ María Eugenia Rodríguez Vásquez
}

Recibido: 19 de agosto de 2016 - Aprobado: 19 de octubre de 2016

\section{Resumen}

Los estudios en las ciencias de la organización son más transdisciplinares hoy y se acompasan bajo la teoría de la complejidad, dada su dinámica. El presente artículo aborda dichos estudios desde el discurso managerial y, de manera transversal, desde la cultura. Asimismo, explica la connotación cultural del management y de la burocracia para contextualizar la figura del burócrata y del político en la organización. Luego de configurar los ideales de tipo weberiano, analiza el paradigma organizacional de la burocracia y del new public management, para posteriormente revisar y develar el estado de la literatura del objeto de estudio mediante el análisis de contenidos - técnica de los métodos cualitativos.

Palabras clave: cultura organizacional, burocracia, administración pública, nueva gestión pública, análisis de contenidos.

Artículo de reflexión. El documento surge del análisis de la cultura organizacional y no está adscrito a ningún grupo o proyecto de investigación. DOI: http://dx.doi.org/10.15332/s0120-8454.2016.0090.05

* Economista y magíster en Políticas Públicas. Candidato a PhD en Administración de la Universidad del Valle. Profesor de Políticas Públicas del Programa Académico de Estudios Políticos y Resolución de Conflictos, adscrito al Instituto de Educación y Pedagogía (IEP) Univalle. Investigador de los grupos: Derecho, Sociedad, Estado y Gestión, y Políticas Públicas, adscritos a la Facultad de Ciencias de la Administración, Universidad del Valle. Correo electrónico: rafael. vergara@correounivalle.edu.co

** Joven investigadora. Asistente de investigación en políticas públicas. Unidad de Apoyo en el Concejo de Cali. Profesional del Programa Académico de Estudios Políticos y Resolución de Conflictos. Instituto de Educación y Pedagogía (IEP), Universidad del Valle. Correo electrónico: maria.rodriguez.vasquez@correounivalle.edu.co 


\title{
Study of the literature review about organizational culture in the Public Administration from a content analysis approach*
}

\author{
Rafael Vergara Varela** \\ María Eugenia Rodríguez Vásquez ${ }^{* * *}$
}

\section{Abstract}

Nowadays, the studies on the organizational sciences are more transdisciplinary, all of them under the theory of complexity, given its dynamics. This article addresses these issues from the managerial discourse and from the culture. It also explains the cultural connotation of management and bureaucracy to contextualize the figure of the bureaucrat and the politician in the organization. After configuring the Weberian ideals type, it analyses the organizational paradigm of the bureaucracy and the new public management, to subsequently examine the literature review of the research object.

Keywords: Organizational culture, bureaucracy, public administration, new public management.

Reflection article. The document results from the analysis of the organizational culture and is not attached to any research group or project.

* Economist and master in Public Policies. PhD candidate in Administration of the University of Valle. Professor of Public Policies of the Academic Program of Political Studies and Resolution of Conflicts, attached to the Institute of Education and Pedagogy (IEP) Univalle. Researcher of the groups: Law, Society, State and Management, and Public Policies, attached to the Faculty of Administration Sciences, University of Valle. Email: rafael.vergara@correounivalival.edu.co

Young researcher. Research assistant in public policies. Support Unit in the City Council of Cali. Professional from the Academic Program of Political Studies and Resolution of Conflicts. Institute of Education and Pedagogy (IEP), University of Valle. Email: maria.rodriguez.vasquez@correounivalival.edu.co 


\title{
Étude de l'état de la littérature de la culture organisationnelle dans l'administration publique depuis le point de vue de l'analyse de contenu*
}

\author{
Rafael Vergara Varela** \\ María Eugenia Rodríguez Vásquez ${ }^{* * *}$
}

Résumé

Les études sur les sciences de l'organisation sont plus transdisciplinaires aujourd'hui et se regroupent sous la théorie de la complexité, étant donnée sa dynamique. Cet article aborde ces études depuis le discours directif et, de manière transversale, depuis la culture. De la même manière, il explique la connotation culturelle du management et de la bureaucratie pour contextualiser la figure du bureaucrate et du politique dans l'organisation. Après avoir configuré les idéaux de type wébérien, il analyse le paradigme organisationnel de la bureaucratie et du new public management, pour ensuite réviser et révéler l'état de la littérature de l'objet d'étude au moyen de l'analyse de contenus - une technique des méthodes qualitatives.

Mots clés: Culture organisationnelle, bureaucratie, Administration Publique, nouvelle gestion publique, analyse de contenus..

Article de réflexion. Le document surgit de l'analyse de la culture organisationnelle et il n'est assigné à aucun groupe ou projet de recherche Article de réflexion. Le document surgit de l'analyse de la culture organisationnelle et il n'est assigné à aucun groupe ou projet de recherche DOI: http://dx.doi.org/10.15332/s0120-8454.2017.0090.05

* Économiste et magister en Politiques Publiques. Candidat PhD en Administration de l'Université del Valle. Professeur de Politiques Publiques du Programme Académique d'Études Politiques et Résolution de Conflits, assigné à l'Institut d'Éducation et de Pédagogie (IEP) Univalle. Chercheur des groupes: Droit, Société, État et Gestion, et Politiques Publiques, assignés à la Faculté de Sciences de l'Administration, Université del Valle. Email: rafael.vergara@correounivalle.edu.co

** Jeune chercheuse. Assistante de recherche en politiques publiques. Unité d'Appui dans le Conseil municipal de Cali. Professionnelle du Programme Académique d'Études Politiques et Résolution de Conflits. Institut d'Éducation et de Pédagogie (IEP), Université del Valle. Email : maria.rodriguez.vasquez@correounivalle.edu.co 


\section{Introducción}

Para la identificación del sujeto y su relación con la cultura organizacional, la conceptualización ontogenética y filogenética del hombre es fundamental para reconocer una sustancia común (polity) que genere en la sociedad valor público (values) sobre la idea del contrato social que identifica a la política (politics). Esta última, como campo de acción, representa una red de relaciones en torno al poder y a las actividades del Estado, donde participan diversos actores con diferentes roles; gran parte de estos conforman las filas de la burocracia pública.

Así, hay dos tipos de sujetos que crean e imponen cultura en las organizaciones públicas: uno burocrático, que integra la administración pública y vive de la política, y el otro, político, que puede vivir o no, para y de la política, siendo semiprofesionales. Ambos plantean una conceptualización de ética-moral, en la que expresan el deber ser de su función; respondiendo dentro del modelo de cultura a diferentes formas de pensamiento lo cual es necesario en el debate sociológico institucional contemporáneo que introduce un método de abordaje a los estudios de la cultura en la organización pública.

Empero, hay que ilustrar a través del documento hacia dónde se contextualizan los estudios de la organización y posteriormente mostrar los resultados desde el método cualitativo en el enfoque del análisis de contenido (Bardin) en el estado de la literatura del campo de la administración pública en el cual transversalmente la categoría cultura organizacional devela unas dinámicas, un enfoque o unas tendencias en este campo de estudio disciplinar.

\section{El discurso managerial y su historia, un concepto integral: organización y cultura}

La burocracia es un término reciente en comparación con el sujeto político, que aparece desde los atisbos de la modernidad, que, a juicio de diferentes autores, se ubica el desarrollo de Occidente en Grecia. Es decir, la modernidad ateniense expone al sujeto que se reafirma en su individualidad (Vernant, 2001, p. 13). Además, nuestras formas de pensar responden a las reglas del lenguaje y las formas que nos gobiernan, todo es producto de la antigüedad, de hecho, Jenofonte hablaba implícitamente de la administración como una forma de instruir sobre la dirección eficiente y el liderazgo (Gomperz, 1955, p. 528)

La sociedad griega es igualitaria, no tiene castas; ni religiosa, ni militar. La urbe o la polis integra a sus ciudadanos en un mismo plano salvo a los esclavos que están por fuera de la humanidad. En Grecia el sujeto se integra a lo religioso, lo político y lo social. Esto significa que es un agente sujeto de derecho, político y persona privada. Por lo tanto, el ciudadano griego es sujeto político que actúa, delibera y participa en el ágora (Vernant, 2001: 15). Desde lo político, el sujeto 
de la polis vive un mundo de iguales, siempre y cuando se le considere al otro ciudadano.

Basta recordar que la sociedad griega planteo dos ideas de individualismo. Una determinada por el gobernante autoritario, con poder para tomar decisiones administrativas para el interés social. La otra determinada hacia el desarrollo del ciudadano individual, quien tomaba decisiones. Estos dos conceptos contribuyeron a la formación de la sociedad griega sobre la idea de "la administración".

Posteriormente en el mundo feudal el individuo en la sociedad política es un sujeto restringido en derechos, pues el orden social se define entre señores y siervos atados a un feudo. Desde el enfoque Eurocentrista, el orden implica un llamado al mundo, la civilización y al reconocimiento del individuo; un llamado al orden de las instituciones (una disciplina común). El orden feudal es autoritario, controlador y de exclusión social en relación al poder, además orgánicamente se desarrolla bajo un esquema piramidal en relación a sus jerarquías, también por eso su mundo se edifica cerrado por medio de fortalezas que sirven para protegerse de las agresiones del enemigo externo (Duby, 2001, p. 528).

La naturaleza del individuo político se evidencia al desarrollarse el mundo económico porque a partir de allí se generan las nuevas reglas del juego para la sociedad. Instituciones que regulan y reconocen la participación del sujeto como ciudadano político y económico en la sociedad (Homo economicus), en la medida en que se genera la transición del feudalismo al capitalismo (Dobb, 1971, pp. 468-480). Es decir, cuando la autoridad y el poder dejen de ser de carácter tradicional por herencia patriarcal a una sumisión que se categoriza racional (en función de medios afines).

Desde el origen del capitalismo el hombre se convierte en "un ser material por naturaleza, es decir una mercancía" (Marx), instalándose en el nuevo universo de los grandes metarrelatos, como el progreso, el ascetismo, la ganancia, el bienestar, la justicia y la igualdad, sustentado todo a través del lenguaje, la razón y la cultura (Agamben). Ahora, lenguaje y cultura son el objeto de instrumentalización del mundo, donde el hombre sale del arquetipo feudal y se instaura en la transformación material del mundo (materialismo histórico de Marx), a través del cual, la especificidad humana evoluciona del taller artesanal donde lo hace absolutamente todo, a la transición de la producción en masa bajo las nuevas reglas de la división del trabajo.

Asimismo, se genera una transformación cultural dialéctica, mediante reglas que permitieron esos grandes cambios, convertidos en relatos duales como campo/ciudad, religión/trabajo, ricos/pobres, feudalismo/capitalismo, estado/ nación, obreros/capitalistas, amo/esclavo (Hegel), nobleza/clase social, taller/ fábrica, entre otros. Es un nuevo orden social donde el sujeto queda atrapado 
en los esquemas de la industrialización (iron cage ${ }^{1}$ ), en una jaula de hierro, al mejor estilo de Kafka en su relato La metamorfosis: "Cuando Gregorio Samsa se despertó una mañana después de un sueño intranquilo, se encontró sobre su cama convertido en un monstruoso insecto". Este nuevo sujeto, que también es organizacional, es un sujeto atrapado, burocrático kafkiano, pragmático, instrumental.

\section{Management y burocracia, una sola estructura y dos actores: el burocrático y el político}

Los orígenes de la estructura epistemológica del management se remontan a casi un siglo, tanto en Norteamérica como en Europa. De hecho, la estructura directiva primigenia es, en esencia, bastante pragmática, en función de su instrumentalización, pues en su conceptualización como en su estructura metodológica fue descrita y hecha por ingenieros en un esquema tecno-científico de prácticas productivas para un fin específico dentro de una cadena productiva en una planta o taller, en un sistema industrial o una organización empresarial, prueba de ello es el corpus argumentativo descrito en Taylor, Ford y Fayol a través de lo que se denominó el primer management; tratados de administración científica.

En el libro; "la administración entre la tradición y la renovación" de Omar Aktouf se distingue que la organización racional del trabajo toma en cuenta a autores como Smith, Babbage y Taylor. Para 1776, Smith describió el desarrollo de la organización empresarial de lo micro a lo macro, trazando el rumbo de la industrialización de Occidente. Sin embargo, el complemento a la racionalización de la división del trabajo en la organización lo aportó Babbage sobre la base de la subdivisión con mayor profundidad, medición y precisión.

Es por ello que el equilibrio en el management es "statu quo"; lo que significa que es funcionalidad del sistema, del mercado, de las lógicas poder; es bastante discursivo. En ultimas es un equilibrio de carácter organicista-homeostático (dado que el funcionalismo era una ingeniería gradual de ajuste societal), básicamente los dispositivos del management son pragmático-universales y apuntan a cuestiones como la eficiencia, la eficiencia-eficacia, tras los tópicos contemporáneos de competitividad, innovación, productividad y rentabilidad, es decir, como plantea Omar Aktouf, "el management es un brazo armado del economicismo" (ejemplo de ello es el marketing). En últimas, la eficacia económica directiva sigue siendo dominante.

Todo pensamiento es conciencia y es acción, es decir se debe separar la decisión de la acción, en la retórica de la gestión pública; los Directivos (o Gobernantes)

1 El concepto iron cage fue interpretado por Talcott Parsons, en la traducción de La ética protestante y el espíritu del capitalismo de Weber, de la noción de stah/hartes Gehäuse (carcasa dura como el acero), lo cual hace referencia a una estructura racional, objetiva y rígida que condiciona el accionar de los sujetos. Véase Gros, A. (2015). Burocratización y racionalización en Max Weber a la luz de las interpretaciones actuales de su obra. Revista Questión, 1(45). Recuperado de http://sedici.unlp.edu.ar/bitstream/handle/10915/44790/Documento_completo.pdf?sequence=1 
"los sujetos políticos" deciden, los mandos medios (burócratas) y bajos de la organización ejecutan (la sociedad legitima la autoridad), en conclusión, es pragmaticismo.

Desde el análisis sociológico organizacional, Max Weber (1995) expresa sobre la vocación del político, describiendo su tipología, surgimiento y posterior evolución del funcionario como figura histórica; el cual tiende a vivir de la política o para ella, siendo esta una empresa de dominación; iniciando una distinción en cuanto a los medios necesarios para trabajar en dicho ámbito. Así, en medio de la clasificación realiza una exposición sobre el proceso de especialización de la burocracia, las características, los medios y fines, que se tienen en cada tipología según el momento histórico al que pertenece; expresando la importancia del poder, al ser este el medio para conseguir ciertos fines; $y$ al ser el fin que pretende conseguir el político, según la tipología a la que pertenezca.

Claro está, no se pasa por alto la relación que plantea en cuanto a la ética y la política, pues, aunque son antagónicas, se debe tener presente que él político ha de actuar, partiendo del deber ser, bajo una ética que plantee lo mejor con respecto a la administración y a las malas prácticas concebidas en esta; sin embargo, el hecho de que tenga intereses específicos y busque, bajo los medios posibles, sus fines, tras vislumbrar sus beneficios, puede hacer que este tienda a salirse de la reglamentación social bajo la cual debe actuar.

En el campo político hay diversos tipos de actores, cuya participación en el escenario difiere en función de sus roles, lo cual lleva a reflexionar sobre a qué responde cada uno de ellos, y cuál es su concepción sobre lo correcto para cada circunstancia, partiendo del hecho de que se encuentran en el mismo campo, preguntándose en el fondo cuál de ellos es el ideal para dicho contexto. Esto debido a que nos encontramos con múltiples posturas en la conducta en el Estado, por lo cual es pertinente hallar un tipo.

La política como campo de acción presenta una serie de diversas interacciones enmarcadas en una lógica que pertenecen a la teoría de los sistemas, partiendo desde el imaginario de David Easton (behaviorista), mediante la cual se interpreta la vida política como un sistema de conducta adaptativo, autorregulador y autotransformador (Cuenca, 1993, p. 133), esta presenta como finalidad la asignación autoritaria de valores, y para ello cuenta con una estructura organizativa.

Dicha estructura se compone por diferentes tipos de sujetos, que desde sus roles pretenden e inciden en la distribución de poder, y la creación de valor público, dentro del cual se enmarca la construcción de una ética organizativa. La ética responde a cada rol de manera diferente, ya que estos se hallan en diferente posición y poseen diversas características, por ello su construcción moral responde a diferentes lógicas.

Partiendo de Weber (1995, p. 92) se encuentran tres tipos de políticos: 1) ocasionales, conformados por todos, al participar de alguna manera de las actividades 
en torno al Estado; 2) profesionales, quienes no desean gobernar por sí mismos, sino que sirven a un jefe político, viviendo de la política; y 3) semiprofesionales, cuyo deseo es desempeñar las actividades solo en caso de necesidad, pues viven para la política.

La categorización weberiana permite vislumbrar dos tipologías de individuos, partiendo de sus roles y funciones: primero, un sujeto burocrático, inmerso en las lógicas organizacionales, con un manual de funciones que determina su accionar, no posee los medios materiales de la administración, y vive de la política. Y segundo, un sujeto político, cuya función tiende a ser de dirección, y no vive de la política sino para ella.

Ahora, la estructura organizativa, o forma de organización, llamada burocracia se plantea como una esfera ética sustantiva, que puede ir en contra de ciertas normatividades sociales, pues se concibe como una expresión parcial de una racionalidad instrumental que solo se sostiene reprimiendo y marginando ( $\mathrm{Du}$ Gay, 2000, p. 59). A la vez, el sujeto político se enmarca en un contexto de dominación, el cual desea poseer y por ello busca por diferentes medios acceder a las diversas capas que conforman el ámbito político, sin importar que para ello tenga que usar todo tipo de medios.

Downs (1950), se logra comprender refinadamente que el dirigente político y el administrador público (burócrata) no son perfectos altruistas ni tampoco filántropos comunitarios dado que no les interesa maximizar ni mejorar el bienestar de la sociedad, ya que a este tipo de sujetos que lideran procesos político-administrativos solo le interesa el dinero, el prestigio y poder que supone gobernar (Downs, 1957, p. 110).

\section{Cultura organizacional. El sujeto burocrático y el político: una descripción}

El sujeto burocrático como constructo de la estructura organizativa, plantea su accionar en el Estado como medio de vida, estando su obediencia a título del poseedor de los medios administrativos; este sujeto ha evolucionado junto con la administración, y así se ha convertido en un funcionario especializado con una clara visión de la política, la cual no ve como lucha por el poder sino como un campo el cual ha de limitarse a administrar (Weber, 1995, pp. 89-115).

La construcción del funcionario responde, por ello, a un personal libre, que se debe exclusivamente a las responsabilidades de su rol y a competencias fijadas. Aquel parte de un contrato sobre la base de la libre selección, y de la calificación profesional, el cual, al vivir de la política, es retribuido con un dinero en sueldos fijos. Así, ejerce su cargo como su principal profesión, se plantea una carrera administrativa, separada de los medios administrativos y sin apropiación del cargo, y está sometido a una rigurosa disciplina y vigilancia (Weber, 1964, p. 176). 
Al encontrarse dicho individuo inmerso en la organización, es fundamental, más allá de describirlo, definir las características de la organización, que fomenta formas de conducta y ámbitos de acción que configuran la moralidad organizativa y, por ende, la noción ética que debe guiar al funcionario. Partiendo de esto, se encuentra que la burocracia, como forma de organización en la cual el poder se concentra en manos de funcionarios técnicos, plantea entre sus dimensiones una jerarquía de autoridad definida, una división del trabajo basada en la especialización, un sistema de normas que define derechos y obligaciones, un sistema de procesos, una impersonalidad relacional y la selección basada en la competencia técnica (p. 108).

El funcionario, al evolucionar como parte de la burocracia - la cual, en su consolidación, se ha planteado su profesionalización, el principio de transparencia, la descentralización, la desconcentración, un enfoque nuevo de control, un aparato flexible y un Estado orientado a la ciudadanía, con normas a favor de evitar la incertidumbre y lo imprevisible en sus procesos y en el contexto cambiante que se les presenta a diario (Mejía, 2005, pp. 3-9) - ha configurado en su rol atributos éticos como la adhesión a los procedimientos, la aceptación a la obediencia y la autoridad, y el compromiso con los fines de la función pública (Du Gay, 2000, p. 62).

Partiendo de lo anterior, en este individuo la ética es el resultado de técnicas y practicas mediante las cuales los funcionarios configuran su conducta para cumplir con su función pública (p. 62). Así, se encuentra que el burócrata ante las circunstancias responde con imparcialidad, con base en su conocimiento profesional y obediencia a sus superiores; su deber no es otro que cumplir con el manual de funciones interpuesto por la organización, sin importar si sus opiniones difieren con él, pues debe limitarse a separarlas de su función y ser fiel a la implementación dichas normas (Mouzelis, 1973, p. 27), configurando así una ética burocrática, la cual responde a una serie de reglas procedentes desde arriba, las cuales plantean lo correcto para su función.

Ahora, el sujeto político, hace referencia a un cuadro dirigente, directivo; de políticos semi-profesionales, independientes, que no viven directamente de la política sino para ella, los cuales pretenden hacer política y están dispuestos a usar diversos medios que poseen para ello. Estos desean el honor de asumir personalmente la responsabilidad de todo lo que se hace (Weber, 1995: 115), con el fin de que la organización perdure.

Con base en Maquiavelo (1513), el sujeto político puede ser conceptualizado como el príncipe, quien debe tener el favor de sus súbditos (burocracia/funcionarios) y acercarse a ellos desde diversos ámbitos (cap. 3), con el fin de que los procesos se realicen adecuadamente; también debe asegurar su autoridad y reconocimiento (cap. 4), designando así en la estructura burocrática algunos cargos con cierto poder sin abandonar del todo su mandato; por otro lado, los actos de severidad siempre deben aumentar (cap. 8), debe hacer buen uso de las leyes y la fuerza, estar dispuesto a actuar según lo exijan las circunstancias, no 
apartándose del bien sino sabiendo hacer el mal cuando es necesario (cap. 18) tomando estos actos y el mal necesario en el contexto como acciones de control sobre los funcionarios, castigos, memorandos, etc.

Ahora, la división de trabajo en la burocracia lleva a que el sujeto político pueda dejar a otros los hechos odiosos, y reservarse los actos de gracia y gloria; estimando a los grandes, y no haciéndose aborrecer del pueblo ni de la clase poderosa (Maquiavelo, 1513, cap. 19), así, cualquier error cometido no caerá en primera instancia sobre su cabeza sino sobre un funcionario delegado, el cual por la caracterización de su rol se verá claramente comprometido. Sin embargo, para que ello ocurra fácilmente, el sujeto político debe aprender a darse a estimar, a declararse amigo o enemigo, debe apoyar los talentos y honrar a sus subalternos que sobresalgan (cap. 21); de esta manera, llega a saciar la ambición de quienes le siguen en la burocracia, y les inscribe en su moral el pensar primero en él y en la organización (cap. 22).

Debido a lo anterior, la ética en este sujeto se plantea como finalidad la consecución de un valor último: el poder representado en la gloria, en la dirección de la política, de las estructuras, que es el fin político. Así, para su mantenimiento, se aconseja que el dirigente o el sujeto político debe tener ausencia de prejuicios, el uso del poder político sin ningún tipo de escrúpulos morales; con ello no quiere decir que debe alejarse del bien, por el contrario, debe transitar por el bien y cada que sea necesario debe incurrir en actos de maldad, de esta forma el uso del mal no es simplemente un hecho sino una necesidad de lo político, planteando actos de maldad, como acciones de disciplina y control sobre la burocracia.

\section{Paradigma organizacional: de la burocracia a la nueva gestión pública y las políticas}

Las nociones de Estado y política pública están plasmadas en proyectos planificados a largo plazo en países institucionalizados. No obstante, en Latinoamérica dicha concepción es una simple "utopía", la cual quisiera acercarse a la realidad. Según North, esto es debido a que no han experimentado fases de desarrollo político-institucional, con similares características al de los países desarrollados. Ahora bien, muchos de los investigadores en políticas públicas concuerdan que el Estado es una institución que rige y ejecuta unas reglas del juego dentro de una sociedad.

Por ello, se establece que las políticas públicas son programas de acción representados a través de decisiones realizadas por el Estado, cuyo propósito es instaurar unas reglas del juego operantes. Todos estos conceptos nos llevarían a plantear que una política pública se realiza cuando las "instituciones" asumen la tarea de satisfacer las necesidades sociales para transformar un estado de cosas problemáticas. Por lo tanto, las instituciones son un factor esencial para el desarrollo de la acción colectiva o de las políticas públicas. 
En este sentido, el neoinstitucionalismo se preocupa más por las relaciones de interdependencia entre instituciones sociales y políticas relativamente autónomas, teniendo en cuenta factores exógenos como creencias, paradigmas, culturas, tecnologías y saberes. Este concepto nos puede servir de metodología y herramienta analítica para mejorar la capacidad productiva, el funcionamiento político y, en general, las condiciones de vida de una sociedad.

Por otra parte, el concepto de gerencia pública ha sido parte de la disciplina de la administración pública que ha enriquecido el dominio de su argumentación científica, a través de modelos gerenciales y administrativos aplicados. Sin embargo, ha provocado un singular debate entre académicos, a tal punto de evolucionar entre paradigmas.

Prueba de ello, es la revolución gerencial de 1980, que reacciona a la crisis del Estado de Bienestar, transformando el paradigma burocrático desde el punto de vista económico, político y administrativo, pasando de la cultura burocrática a la cultura gerencial (Martínez, 2002, p. 17). En últimas, esta visión plantea que en nuestros pueblos se debe superar el populismo y el clientelismo de la función pública para reemplazarlo por un Estado de derecho en la búsqueda del buen desempeño de las funciones del Estado.

Por ello, en términos de los paradigmas, se plantea la crisis del Estado en función del paradigma burocrático, es decir, el modelo del Estado de Bienestar hacia mediados de los años setentas hace crisis. Esta visión anquilosada de la intervención estatal, o Estado benefactor, acentúa la crisis del fordismo global, donde la visión gerencial del estado se planteaba bajo la lógica clientelista que supuestamente actuaba dentro de un marco social.

Ahora bien, la globalización del capitalismo de mercado introduce términos como las ventajas competitivas, cuya operación se dirigía hacia la transnacionalización del capital y la infiltración de modelos liberales hacia la misma estructura del Estado, bajo la égida de la eficiencia y eficacia desde el punto de vista institucional.

Por ende, retrotrayendo el análisis desde la óptica de las Políticas Públicas, se diría que las instituciones son las reglas del juego que determinan o guían la conducta de los agentes, los que reaccionan de alguna manera maximizadora para sus propios intereses. Por esta razón, hay que tener en cuenta también que las instituciones pueden ser formales y estar escritas en la constitución, las leyes y los organigramas de las empresas, o ser informales como las normas, las ideologías y las religiones que se constituyen en guías de acción de los agentes sean estos económicas, sociales o políticas (Kalmanovitz, s. f., p. 4).

Para Douglas North, las instituciones están correlacionadas con la dinámica económica. Las políticas institucionales y los cambios políticos son influyentes, ya que actúan para acelerar o frenar el sistema económico. Dado esto, las instituciones determinan los costos de transacción, como de transformación, lo que 
indica, la posibilidad de realizar actividades socioeconómicas, que beneficien o maximicen el bienestar social.

Al finalizar los años setenta se erige un nuevo orden institucional alrededor de la cultura gerencial-administrativa publica anglosajona, donde países como Canadá, Australia y Nueva Zelanda edificaron un nuevo modelo competitivo de gestión, implicando que la gerencia, sus políticas y formas de gestión constituyeran un icono trascendental a escala mundial. Hacia finales de la década de los ochenta, el nuevo modelo de gestión denominado la nueva gerencia pública se concibe como opuesto de la administración pública weberiana, y más particularmente, se le entiende como una transdisciplina que puede sustituirla eficazmente, planteando que la administración del Estado representa un nuevo paradigma pos-burocrático, en el cual la administración es considerada un negocio de orden económico que busca satisfacer al usuario-cliente en una lógica de mercado (Martínez, 2002, p. 19).

La nueva gerencia pública, como visión mercantilizada de lo público, distanciada de los supuestos del management weberiano, tiene el origen en otro paradigma obviamente legitimado bajo la visión del neo-institucionalismo, de la economía, del nuevo institucionalismo administrativo (Powel y Dimagio), la política y del pensamiento económico neoclásico engendrado en la escuela austríaca, así como de la teoría de la elección pública estadounidense, que exalta como factores culturales; lo privado, el individualismo y la rentabilidad. Por lo tanto, aunque la nueva gerencia ostenta a modo de apellido el vocablo público, esta versión no tiene ninguna aproximación de tipo social subsidiario, pues sus resultados tienden a la búsqueda de la privatización del Estado.

En esencia, en las alternativas de cambio de la nueva gestión se encuentran como cultura organizacional; reducir las funciones y tamaño del Estado (reingenieríareformas), eliminar déficits y controlar gasto público (accountability), enfocar a la administración pública en torno a funciones endoprivatizadoras basado en la lógica del mercado (outsoursing). Al mismo tiempo se enfoca en la relación costo-beneficio. Por lo tanto, se trata de un modelo liberal de corte radical bajo las lógicas del mercado.

Es decir, es mucho más pragmática y economicista, por ello se plantea que los individuos tienen una racionalidad limitada. Asimismo, que las instituciones son mecanismos desarrollados para disminuir los costos de transacción en todo intercambio. Esto significa, que este modelo es una rutina institucional para disminuir los costos entre los grupos de presión y los hacedores de políticas públicas. De igual modo, el modelo de la Nueva Gestión Publica coloca unas restricciones al comportamiento basado en el interés personal, o sea limitan las estrategias de los grupos de presión y de los políticos.

Por ello, esta nueva gerencia representa la correlación entre la economía, la administración y la política. Esto se observa, a través de sus conceptos como: orientación al cliente, privatización, mercado y competencia. De igual modo, dentro de 
la reforma neo gerencial del sector público las nociones son: enfoque empresarialgerencial, gerencia por objetivos y resultados como agenciación.

En últimas, lo que anima a la nueva gerencia pública es la reducción de costos. Sin embargo, estas políticas de ajuste estructural terminan siendo "una camisa de fuerza" para quienes integran el sistema organizacional. Es decir, en el modelo hay que calificar en aras de la eficiencia. Si bien, la profesionalización (en teoría) garantiza un desarrollo adecuado para el sector público, pero ello no significa asegurar el éxito a largo plazo. Esto se explica también porque el exceso de profesionalización terminara siendo incompatible con el desempeño de cargos técnicos o de segundo rango en la estructura misma del Estado. Sin embargo, era necesario delimitar las funciones del Estado de manera gradual, y no de manera desbordada como se realmente se aplicó.

Contrariamente a lo que plantea, el sistema es muy permisivo y los pagos terminan siendo iguales o similares a los del Estado de Bienestar, o burocrático sin poco castigo. Es decir, desde lo institucional, los niveles de corrupción no tienen penas altas, es más, el robo o el desangre continuo de depredación del Estado, se realiza en cantidades exorbitantes y termina enriqueciendo al burócrata timador.

Las reformas de gerencia pública terminaron siendo adaptadas y cooptadas por la filosofía de los entes multilaterales, como el Banco Mundial y el Fondo Monetario Internacional - los cuales, dentro de sus recetas, terminaron imponiendo políticas de choque fiscal y monetario, donde se realizaron incrementos en la estructura impositiva, los cuales terminaron siendo impuestos al conjunto de la sociedad, que, en últimas, es el 'paganini' del mismo aparato burocrático.

Asimismo, la política monetaria y el discurso de los bancos centrales, como también de los ministerios de hacienda, con el objetivo de disminuir la inflación, terminan retrotrayendo la capacidad de compra de los individuos, por lo que la excusa de la "inflación" termina convirtiéndose en un impuesto más, amparado bajo la lógica del sistema.

Al respecto, la eficiencia en el sistema de información termina apuntando a la ampliación de la estructura impositiva del Estado, es decir, eludir y evadir impuestos no sería una tarea fácil. En últimas, el modelo y la gestión de desempeño terminan siendo una ilusión, ya que limita al funcionario a que se adecué a unos recursos escasos y a una normatividad drástica, que solo le permiten hacer milagros en términos de resultados e impactos.

Ahora bien, en América Latina la revolución gerencial fue el marco de los procesos de modernización del Estado en los años noventa. Por esto, la primera fase busco orientar nuevas fronteras funcionales con la sociedad, reduciendo el tamaño del Estado, relacionando la apertura exterior, la liberalización económica y la instauración de una filosofía liberal contrariamente a la realidad de los países donde se ha implementado el modelo (Oslack, 1997). Sin embargo, hoy la administración pública debe diseñar alternativas diferentes o bien distintas, 
a las que aquí se han planteado. Es cierto, que existe un mercado promisorio para la producción y venta de bienes públicos pero realmente poco contribuye a la discusión teórica o practica del modelo. Ello indica, que se debe considerar un patrón de interacción entre política y administración que tenga en cuenta los requisitos de inserción social y de regulación política en la búsqueda del accionar social (Martínez, 2002, p. 28).

No obstante, el modelo vislumbro en esencia una imagen orientada a prestar servicios a unos clientes que son los ciudadanos. Por ello, el ideario del management público situó un servicio cuyo cliente era el público, es decir con capacidad de respuesta a la población servida. En este sentido, la tendencia del deber ser de la gestión pública se dirigió a la erradicación del despilfarro y la reducción de los servicios, bajo el precepto paradigmático de las tres E: eficiencia, eficacia y economía. En este orden de ideas, hay que tener en cuenta que la eficiencia debió involucrarse a través del impacto que las políticas generaban en la comunidad transformando el bienestar de la sociedad (bajo el concepto de la eficiencia adaptativa), es decir, donde el impacto de las políticas y de la gestión se corresponda hacia las respuestas de los ciudadanos (Guerrero, 1999).

Por ello, la reforma de la gestión pública apunto a la búsqueda de la eficiencia técnica en torno a una estructura económica. Empero, la gestión pública a futuro debe rediseñar nuevos esquemas y modelos dentro de la lógica de las políticas públicas escandinavas o del bienestar. Entre estas nuevas visiones se debe tener en cuenta las relaciones entre la organización y el entorno social. Con esta visión, las organizaciones públicas desarrollaran objetivos y prioridades, en lugar que las políticas vengan diseñadas por los tecnócratas. En esta propuesta debe quedar claro que más allá de administrar los asuntos públicos, más allá de buscar el modelo ideal, el debate debe actual debe centrarse en la racionalidad que busca armonizar la gestión con una clara visión del mundo y del individuo moral como fin último en la búsqueda del bienestar social. En últimas, la gestión pública es un proceso en el que diferentes organizaciones comparten intereses para gestionar el cambio estructural en pro de metas 'socialmente' alcanzables.

\section{Metodología}

Dentro del campo de estudio de los métodos cualitativos, el análisis de contenido es una técnica que permite analizar, clasificar y revelar información oculta que una simple lectura no explicitaría (Berelson, 1952). Basta agregar, además, que esta técnica combina procesos de análisis cualitativos en relación con temas, frases o palabras que, al identificarlos, codificarlos y registrarlos, los ubica automáticamente en el plano de lo cuantitativo, es decir, permite identificar el sentido objetivo de un texto. En relación con lo anterior, para el análisis de contenido cobra sentido y significado al exponer de manera objetiva la intencionalidad de un documento (Bardin, 2002). 
Respecto a la técnica de análisis de contenidos, este aparte busca seleccionar, presentar y analizar el estado de la literatura en relación con los documentos escritos sobre la cultura organizacional en la administración pública. Frente a la dispersión de información, se decidió buscar en una página especializada en administración pública, como la del Centro Latinoamericano de Administración para el Desarrollo (Clad), que estudia las dinámicas y la evolución de la administración pública latinoamericana, y las integra a través de documentos o ponencias que se exponen en los congresos anuales. Por ello, el objetivo de la revisión bibliográfica, más que presentar y dar cuenta de los documentos, busca clasificar e identificar, a través de los contenidos, los posibles escenarios o las tendencias en torno a la categoría cultura organizacional en la administración pública, siendo esta la particularidad y objeto de estudio de los métodos cualitativos en torno al análisis de contenidos (Bardin) (tabla 1).

Tabla 1. Categorías y subcategorías del estudio

\begin{tabular}{|c|c|l|l|l|l|}
\hline \multirow{2}{*}{ Categoría } & Subcategorías & Año & Idioma & País & $\begin{array}{c}\text { Porcentaje de } \\
\text { producción }\end{array}$ \\
\hline \multirow{2}{*}{$\begin{array}{c}\text { Nueva gerencia } \\
\text { pública (cultura } \\
\text { organizacional) }\end{array}$} & $\begin{array}{c}\text { Principios corporativos } \\
\text { (metapolíticas) } \\
\text { Reformas }\end{array}$ & Implementación & & & \\
\cline { 2 - 6 } & Evaluación & & & & \\
\cline { 2 - 6 } & $\begin{array}{c}\text { Estudios de caso sobre } \\
\text { transferencia de políticas }\end{array}$ & & & & \\
\hline
\end{tabular}

Fuente: elaboración propia

Bajo este esquema (tabla 1) se puede plantear y analizar un estado del arte que busca identificar a través de variables o subcategorías, el porcentaje de producción en cada categoría, el idioma, el peso de la subcategoría en la categoría, los países latinoamericanos identificados, los años de referencia en cada categoría. Se debe tener en cuenta, además, que este tipo información no viene discriminado, por lo cual la configuración se establece a criterios de selección de acuerdo a las categorías y a la información relacionada en los documentos base.

\section{Resultados}

Los estudios de la cultura organizacional en la administración pública inician hacia finales de las décadas de los sesenta y setenta, como propuestas de reforma administrativa que critican el modelo de la burocracia, la redistribución 
de poderes, la reforma del servicio civil y la responsabilidad del funcionario público. Institucionalmente, gran parte de la literatura apunta hacia la reestructuración del sector público.

Frente a la literatura se encuentran cuatro subcategorías, enfoques o etapas cronológicas con relación a los objetos de estudio identificados. La primera etapa se puede clasificar como de metapolíticas, porque enuncian elementos propios de la cultura organizacional frente a las reformas establecidas al modelo burocrático weberiano como estructuraciones, reformas administrativas, el desarrollo de la cultura de la evaluación de resultados, comportamientos éticos, cultura gerencial, ética gerencial, liderazgo moral, gerencia ética de las organizaciones.

Tabla 2. Categorías y subcategorías del estudio

\begin{tabular}{|c|c|c|c|c|c|}
\hline Categoría & Subcategorías & Año & Idioma & País & $\begin{array}{l}\text { Porcentaje de } \\
\text { producción }\end{array}$ \\
\hline \multirow{4}{*}{$\begin{array}{l}\text { Nueva gerencia } \\
\text { pública (cultura } \\
\text { organizacional) }\end{array}$} & $\begin{array}{l}\text { (Metapolíticas) } \\
\text { Principios corporativos } \\
\text { Modelos reformas }\end{array}$ & $\begin{array}{l}1979- \\
1991\end{array}$ & $\begin{array}{c}\text { Inglés } \\
\text { Portugués }\end{array}$ & $\begin{array}{c}\text { Bélgica } \\
\text { Filipinas } \\
\text { Inglaterra } \\
\text { Canadá } \\
\text { Estados } \\
\text { Unidos }\end{array}$ & $\begin{array}{c}90 \text { (inglés), } \\
10 \text { (portugués) }\end{array}$ \\
\hline & Implementación & $\begin{array}{l}1990- \\
1991\end{array}$ & $\begin{array}{l}\text { Ingles } \\
\text { Holandés } \\
\text { Portugués }\end{array}$ & $\begin{array}{l}\text { Canadá } \\
\text { Nueva } \\
\text { Zelanda } \\
\text { Estados } \\
\text { Unidos } \\
\text { Brasil } \\
\text { Holanda }\end{array}$ & 99 (inglés) \\
\hline & Evaluación & $\begin{array}{l}1991- \\
1999\end{array}$ & Inglés & $\begin{array}{l}\text { Inglaterra } \\
\text { Estados } \\
\text { Unidos }\end{array}$ & Inglés \\
\hline & $\begin{array}{c}\text { Transferencia de } \\
\text { políticas-casos }\end{array}$ & $\begin{array}{l}2000- \\
2011\end{array}$ & $\begin{array}{l}\text { Inglés } \\
\text { Español }\end{array}$ & $\begin{array}{l}\text { Argentina } \\
\text { México } \\
\text { Estados } \\
\text { Unidos }\end{array}$ & $\begin{array}{l}56 \text { (español) } \\
44 \text { (inglés) }\end{array}$ \\
\hline
\end{tabular}

Fuente: elaboración propia

La gerencia pública, según este enfoque, pretende mejorar sus indicadores basándose en el sector privado, en cuanto a la adopción de una visión general de las metas, cuyo desarrollo y mantenimiento es la base de la cultura organizacional; de igual forma, tiene en cuenta la formación del liderazgo efectivo, que como modelo ético transformaría el carácter amoral de las organizaciones burocráticas promocionando y estableciendo conductas éticas a través de 
códigos y normas aprehendidas mediante un enfoque top-down, aunque cabe aclarar que en el ser dichas reglas de juego pueden solo representar un valor simbólico, para legitimar profesionalmente a la autoridad (Duncan et al., 1991; Kernaghan, 1991; Fischer y Zinke, 1989).

El integrar las técnicas del sector privado a la gestión pública busca aumentar la eficiencia y eficacia, desarrollando y estimulando un sistema de planificación estratégica (PE) para evaluar el rendimiento, tendiendo a las observaciones en prospectiva, con base en las necesidades de los usuarios, y los resultados de las organizaciones. Por lo cual se retoman dos pautas del sector privado: la innovación y las relaciones con los usuarios; con las cuales además suministra seguridad a los empleados, y productos de alta calidad. Claro está, pese a partir de la cultura del sector privado las culturas de gerencia pública se distinguen ya que sus rasgos hacen referencia al valor que en ellas se atribuye al aprendizaje organizacional, a la adaptación, y a la capacidad para dar respuesta flexible ante el surgimiento de nuevos problemas y prioridades (Duncan et al, 1991; Metcalfe y McGill, 1988; Richards, 1987).

Ahora bien, los sujetos de las organizaciones difieren según su cultura organizacional, ya que sus actitudes y creencias en relación con la organización se originan según el sector al que pertenecen, el contexto y otros factores. De esta manera, los gerentes públicos consideran que sus instituciones en los países en desarrollo son innovadoras y responden adecuadamente a los cambios organizacionales. Las tareas se sienten estimulantes, y creen que sus opiniones tienen peso en la toma de decisiones. Sin embargo, simultáneamente, los gerentes de los países en desarrollo confirman las opiniones que estiman que existe un sesgo hacia los intereses personales más que hacia los intereses organizacionales, y que el liderazgo tiene tendencia a ser directivo (Rahman y Norling, 1991).

El segundo escenario revela cómo fueron implementados los modelos de reforma administrativa bajo el new public management. Aquí, el $99 \%$ de los casos muestran que el tipo de publicación se realiza desde países angloparlantes, es decir, se relatan las experiencias de aplicación o ejecución del modelo.

La evolución de este enfoque inicia en la década de los sesenta, con mayor influencia en el sector privado, por lo cual en sus antecedentes teóricos se vislumbran el desarrollo de la gerencia estratégica, la importancia de la creatividad y la innovación, por su utilidad en la gestión pública, para lo cual se tienen presentes los aspectos principales de la actividad administrativa (Vinzant, 1996). La teoría de la cultura organizacional aquí desarrollada deriva de las ciencias políticas tras el declive de la teoría de la escogencia racional, para lo cual se preguntaba por la utilización de la metáfora cultural en el análisis y en la implementación de la reforma administrativa, discutiendo la existencia de aspectos específicos de una cultura administrativa (Keraudren, 1996).

La búsqueda de la definición del rol de la cultura organizacional en el cambio administrativo puede aprovechar una serie de lecciones del pasado en esta 
materia, lo cual es primordial dado que en esta se apoya y desarrolla la nueva gestión pública. La cultura debe entenderse, según Wildavsky, como "valores compartidos vinculados a prácticas sociales", por lo que en el campo de la administración pública, la teoría cultural debe integrar valores y prácticas en un único enfoque que abarque el análisis cultural un conjunto de interacciones complejas y autónomas, partiendo del nivel macro, teniendo presente que la cultura incide en el ámbito interno, y es un factor decisivo en la imagen que la organización proyecta hacia el exterior.

Esta perspectiva cuenta con experiencias de países de la Comunidad Británica en la construcción de una cultura del éxito para la administración pública; y Brasil en cuanto a la estructura y el funcionamiento del Sistema Integrado de Administración Financiera del Gobierno Federal brasileño (Siafi). De las cuales se percibe que las reformas se originaron con el objeto de reestructurar las organizaciones para obtener mayor autonomía gerencial, e introducir sistemas de desempeño tanto a nivel individual como organizacional, así como tratar las problemáticas administrativas en la gestión de recursos por medio del control financiero y la innovación técnica desarrollada para minimizar los costos e incrementar la eficiencia (Kaul, et al. 1995; Roger Dos Santos y Freitas, 1994; Mello Rodrigues, 1994).

El tercer escenario se centra en la evaluación y se ubica en aspectos como la evaluación del desempeño, lenguaje que no existía en el modelo burocrático y que hoy hace parte de la cultura organizacional de la administración pública. La mayoría de los temas sobre evaluación han sido publicados en los países angloparlantes con metodologías adecuadas para cada caso.

Este enfoque plantea un reinvencionismo estatal en diferentes áreas, debido al contexto político y administrativo en el que se desarrolló. Entre sus estrategias está el cambio en la cultura organizacional, con base en los postulados del gerencialismo, dando importancia a temáticas concernientes a la evaluación y el control de resultados. De igual manera, la reinvención pretende no afectar la confianza en la administración, presentando medidas orientadas a dirigir la labor administrativa hacia el ciudadano-usuario de los servicios públicos; a la asignación de atribuciones a los empleados para obtener los resultados deseados; a encarar la pérdida de tiempo y el despilfarro de recursos; e innovar en el trabajo para reducir costos y mejorar la eficiencia; lo cual se puede vislumbrar a través de las evaluaciones (Kamensky, 1996; Gore, 1994).

Entre las experiencias a destacar se encuentra la "Revisión del Desempeño Nacional" en Estados Unidos, del vicepresidente Al Gore; que da cuenta de los postulados anteriormente planteados. Asimismo, al observar otros casos se encuentran comparaciones entre los Estados Unidos y la República Popular China, que parten de tener como objeto común a la cultura los valores subyacentes a los derechos y responsabilidades de los empleados del sector público, con lo cual se encuentra que los aspectos culturales inciden de manera directa e indirecta las concepciones sobre esto, determinando la importancia, el tipo 
de relaciones laborales y contribuyendo con la determinación de la legitimidad de ciertas prácticas, las cuales pueden interpretarse como derecho o responsabilidad, en función de los valores culturales existentes (Gore, 1994; Osigweh y Chimezie; Huo, 1993).

El último escenario, responde a lo que se conoce en la literatura como policytransfer, experiencias o casos. En los últimos 15 años aparecen relatados las experiencias latinoamericanas casi en un $60 \%$ de la literatura existente.

La visión weberiana destaca en este enfoque, donde la Administración Pública es caracterizada por su carácter racional, la aparición de reglas abstractas, el orden impersonal de las decisiones, normas escritas para todo acto, funcionarios con roles diferenciados y formalizados en manuales e instructivos. Estos instrumentos moldearon la cultura propia del aparato público que persiste actualmente, transfiriéndose a los nuevos miembros de la organización como la verdadera forma de conducirse, junto a los cuales es posible observar comportamientos guiados por normas no previstas por el sistema formal, que influyen en la forma de concebir y actuar al interior de la misma: ciertos códigos no escritos, ciertas reglas invisibles (Blutman, 2011).

El cambio en la cultura organizacional de la administración pública, conlleva a la definición del término "cultura", la cual se constituye en todo aquello que permite conocer el mundo al individuo, mientras que por su parte, la cultura organizacional se define como "la personalidad de la organización y comprende el conjunto de valores, mapas mentales, creencias, señales tangibles (artefactos) y actitudes compartidas por los miembros de la organización en un tiempo determinado..." (Gallardo, 2001, p. 87; Martínez, 2004).

Un caso de este enfoque es la administración pública argentina, donde la planeación no tiene el éxito deseado porque no se involucra a la población en el diseño de sus instrumentos, quedando esta función relegada solo a la función y rol de los funcionarios, sin practicarse el consenso social, por lo cual no existe compromiso de la sociedad, ni de las instituciones, sumado a los problemas culturales que prevalecen hasta la actualidad, como el soborno, la extorsión, los fraudes fiscales, entre otros (Orbegozo, 2004), prácticas que llegan a convertirse en una cultura paralela informal arraigada en los sujetos.

Es necesario que la presente administración tenga en cuenta mecanismos de participación ciudadana para la elaboración y evaluación de planes y programas, con miras a un verdadero cambio estructural que vincule a la sociedad con las acciones del Gobierno y genere un sistema basado en los principios democráticos. También debe proveer a la población de especialistas que funcionen como apoyo técnico para una fructífera planeación, que traten de aumentar la escasa motivación de la población para la participación en comités o asambleas vecinales y también lograr la reducción de las resistencias habituales que cada individuo y comunidad tienen al proceso de cambio, ya que muchas veces se sienten afectados en sus intereses personales. 
Gráfico 1. Escenarios en el estado de la literatura.

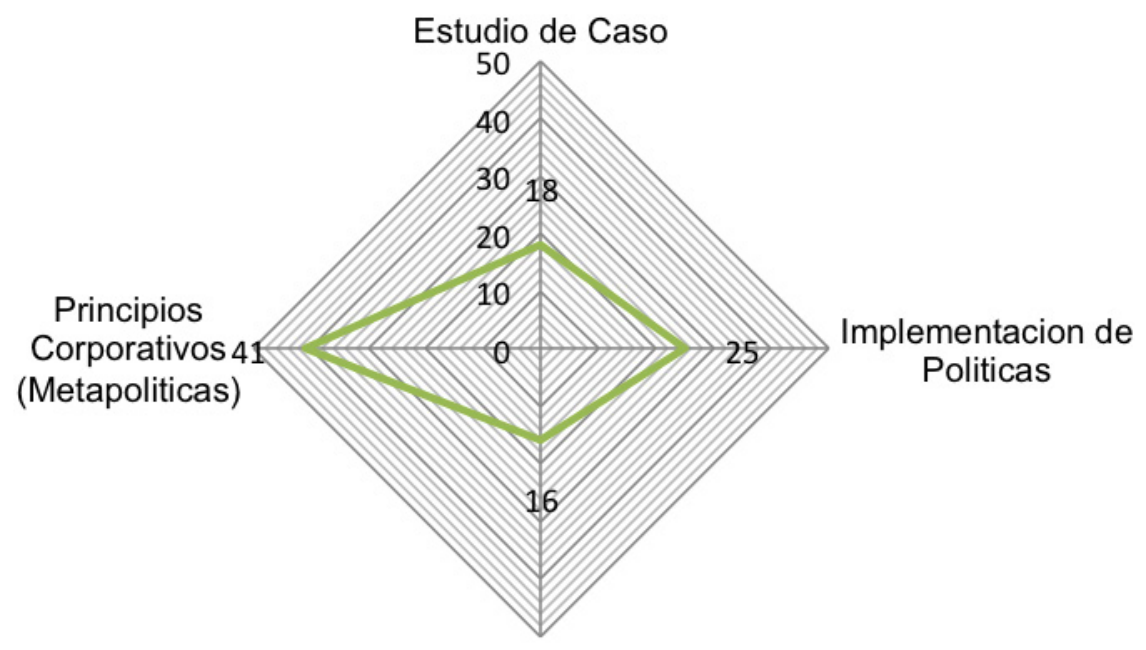

Evaluacion

Fuente: elaboración propia

Finalmente, cuando se hace el análisis por escenarios, se encuentra que en un $41 \%$ se ha privilegiado el estudio por metapolíticas (escenario 1); en segundo lugar, los aspectos referentes a la implementación han tenido interés por describir los efectos culturales en la adopción del modelo gerencial (en un $25 \%$ ), y, en último término, casi en un $20 \%$ la tendencia indica que el objeto de trabajo se presenta sobre casos de cultura organizacional en la administración pública (estudios de caso).

\section{Conclusión}

Retomando nuevamente a (Downs, 1950) este sujeto, político, burócrata y si se quiere "Homo economicus-directivo", producto de la cultura mercantilizada, es hoy un sujeto que ha desmitificado su mundo objetivo para aferrarse fuera del mundo más a sus propias subjetividades, es un sujeto cuya ética es mucho más artificial (por eso se corrompe o lo ve natural hacerlo) además se encuentra más indignado que nunca por sus instituciones, porque la democracia cada vez menos representa los intereses de la sociedad. En últimas es un sujeto líquido, gaseoso, un sujeto en el vacío, desencantado por el incumplimiento de las promesas y de la perdida de los grandes metarrelatos con los que surgió la sociedad moderna (Bauman, 2005).

Hoy este sujeto pasó de los grandes relatos al microrrelato que ordenan su mundo, es un sujeto hedonista, narciso, liquido. Hoy este sujeto es light 
(metrosexual) y se empodera a través los discursos de desmitificar el sentido de la carrera administrativa y de la experiencia. Es un sujeto organizacional cada vez más yuppie (Young Urban Profesional), y sus relaciones humanas, profesionales, empresariales y sentimentales son más liquidas, sin ningún grado de responsabilidad hacia el otro (si y solo si se identifica al otro para afirmar mi Yo). Hoy más que nunca, la sociedad actual, inclusive la neo-burocrática, agencial y organizacional, son verdaderos archipiélagos con sujetos-isla, que se aferran a pequeños relatos, como las tecnologías de información y comunicación, como también a las retoricas del nuevo milenio, con lenguajes psicoorganizacionales como el coaching.

Actualmente la organización en las empresas o en las organizaciones debido a su orden jerárquico conlleva a que existan una serie de diferencias y limitantes entre el subordinado y el que subordina; divisiones que la teoría ha tendido a tratar buscando que sean cada vez menos amplias.

El reconocimiento del "otro" está marcado por el conflicto existente entre el "nosotros" y el "ellos"; el cual genera que se reconozca y legitimen las diferencias entre ambos, ya que la representación de los sujetos viene dada en un enfoque top-down mediante el que la cúspide no se integra del todo con la base, pues esta última es prescindible, y sustituible en cualquier momento. Aunque son motivo de crítica las conductas de tipo autoritario, el trato despectivo, la minimización del otro, etc.; esto no quiere decir que el proceso de humanización del sujeto sea del todo apropiada, además, ni que sea esa la razón por la cual tiene origen, o por otro lado el que la preocupación por el "otro" conlleve a ello; lo cual también hace reflexionar sobre el por qué se da la resistencia al reconocimiento del "otro" o "ellos".

De manera implícita se entiende, que ambos sujetos que responden a sus contextos, ética y funciones se han transformado a lo largo de la historia de la administración publica, de acuerdo a los enfoques descritos anteriormente. Así se conciben sujetos que se preocupan por una serie de elementos diferentes en cada etapa, ya que al evolucionar la administración pública, la cultura organizacional modifica tanto la institución como los individuos que en ella cohabitan.

Los cambios tanto en los sujetos como en las organizaciones tienden a ser cíclicos pasando del aprendizaje del modelo legal racional weberiano a la nueva gestión pública, regresando al inicial, como lo plantea David Arellano Gault (2002) en el artículo "El meteorito que mato al dinosaurio", en el cual deja entender que pese a las reformas implementadas, en los sujetos hay arraigados una serie de reglas de juego que no permite transformaciones absolutas de su cultura. Sin embargo, hay cuestiones que se deben adoptar con el objeto de mejorar los resultados individuales y colectivos, de esta manera, se integra al esquema organizacional la evaluación (accountability), la innovación, la retroalimentación (feedback), etc.

De igual manera ocurre con la burocracia y las políticas públicas, estas últimas como representación del Estado en acción. Las burocracias, como forma de 
organización, íntegra políticas, de acuerdo con los periodos lo planteado en cada enfoque; especialmente lo relacionado a la evaluación de sus resultados, con el objeto de asegurar su estructura y demostrar que cumple con su misión y función, empero hay esquemas y prácticas que no logran flexibilizarse del todo, según el área que se observe, ya que trae consigo tecnicismos y etapas que no permiten aspectos de las reformas como integrar la participación ciudadano o permitir la toma de decisiones en conjunto.

\section{Referencias}

Bardin, L. (2002). Análisis de contenido. Madrid: Akal.

Bauman, Z. (2005). Vidas desperdiciadas. La modernidad y sus parias. Barcelona: Paidós Ibérica.

Berelson, B. (1952). Content analysis in communication research, s. 1: Nueva York: Free Press, Glencoe.

Cuenca, R. (1993). El administrador en el sector público: ¿técnico o político? Cali: Departamento de Medicina Social, Facultad de Salud, Universidad del Valle.

Dobb, M. (1971). Estudios sobre el desarrollo del capitalismo. México, D. F.: Siglo XIX.

Downs, A. (1957). An economic theory of political action in a democracy. Journal of Political Economy, 65(2), 135-150.

Duby, G. (1987). La emergencia del individuo. En Historia de la vida privada. México, D. F.: Siglo XIX.

Du Gay, P. (2000). En elogio de la burocracia. Madrid: Siglo XXI.

Duncan, J., Ginter, P. M. y Capper, S. A. (1991). Excellence in public administration: Four transferable lessons from the private sector. Public Productivity E Management Review, 14(3), 227-236.

Sullivan, T. (Septiembre de 2011). Embracing complexity. Harvard Business Review.

Fischer, F. y Zinke, R. (1989). Public administration and the code of ethics: administrative reform or professional ideology? International Journal of Public Administration, 12(6), 841-854.

Gomperz, T. (1955). Greek thinkers: A history of ancient philosophy (vol 1). Nueva York: Humanities Press.

Hernández, C. y Guerrero, G. (1999). Hacia un nuevo concepto de gestión pública. Innovar. Revista de Ciencias Administrativas y Sociales. (14). URL: http:// www.revistas.unal.edu.co/index.php/innovar/issue/view/2281 
Kaul, M. y Collins, P. (1995). The transition in outlook. Building a culture of success: an overview, 15(3), 257-262. DOI: 10.1002/pad.4230150314.

Keraudren, P. (Enero de 1996). In search of culture: lessons from the past to find a role for the study of administrative culture. 9(1), 71-98. DOI: 10.1111/j.14680491.1996.tb00234.x.

Kernaghan, K. (Marzo de 1991). Managing ethics: complementary approaches. Canadian Public Administration, 34(1), 132-145. DOI: 10.1111/j.1754-7121.1991. tb01447.x.

Kamensky, J. (Mayo-junio de 1996). Role of the "reinventing government" movement in federal management reform. Public Administration Review, 56(3), 247-255. DOI: 10.2307/976448.

Maquiavelo, N. (1999). El Príncipe (1513). Bogotá: Panamericana.

Martínez, E. (Enero-junio de 2002). La revolución gerencial en la gestión pública. Innovar. Revista de Ciencias Administrativas y Sociales, (19). URL: http:// www.revistas.unal.edu.co/index.php/innovar/issue/view/2264/showToc

McGill, R. (Octubre de 1988). Planning for strategic performance in local government. Long Range Planning, 21(5), 77-84. DOI: 10.1016/0024-6301(88)90108-2.

Metcalfe, J. y Richards, S. (1987). Evolving public management cultures. Ponencia presentada en el European Consortium for Political Research Joint Sessions, Barcelona.

Mejía, G. (Noviembre de 2005). Gestión pública: una visión desde el mercado. Revista Inalde. Escuela de Dirección y Negocios. (15), 64-73. Recuperado de http://intellectum.unisabana.edu.co/bitstream/handle/10818/25222/revista_inalde_15.pdf?sequence $=1 \&$ isAllowed $=y$

Mouzelis, N. (1973). Organización y burocracia. Barcelona: Edicions 62.

Oslak, O. (1997). Estado y sociedad: ¿nuevas reglas del juego? Revista Clad: Reforma y Democracia, (9). Recuperado de http://siare.clad.org/revistas/0029802.pdf

Rahman, S. y Norling, F. (1991). Managerial thinking: A study of public managers from developing countries. DOI: 10.1002/pad.4230110203.

Roger Dos Santos y Freitas, H. (1994). O sistema integrado de administracao financeira do Governo Federal (Siafi): instrumento de controle social. RS: Série Documentos para Estudo, No 10/94 PPGA/UFRGS. Porto Alegre: s. e.

Weber, M. (1995). La política como vocación. En El político y el científico (pp. 81-179). Barcelona: Atalaya. 
Weber, M. (1964). Economía y sociedad. México, D. F.: Fondo de Cultura Económica.

Vernant, J. P. (2001). El individuo en la ciudad. En El individuo, el amor y la muerte en Grecia. Barcelona: Paidós.

Vinzant, D. (1996). Strategic management and public organizations: lessons from the past and prescriptions for the future. International Journal of Public Administration, 19(10), 1743-1779. Recuperado de http://dx.doi. org/10.1080/01900699608525164. 